\title{
EN2 as an oncogene promotes tumor progression via regulating CCL20 in colorectal cancer
}

\author{
Yimin Li', Jiaxin Liu', Qing Xiao', Ruotong Tian², Zhengwei Zhou², Yaqi Gan', Yuanyuan Li', Guang Shu² and \\ Gang Yin (1) ${ }^{1,3}$
}

\begin{abstract}
Engrailed-2 (EN2), a member of the engrailed homeobox family, has been shown to be abnormally expressed in a variety of cancers. However, the expression and the clinical significance of EN2 in colorectal cancer (CRC) are largely unknown. Firstly, we found that EN2 acted as an oncogene in CRC. EN2 was upregulated in colorectal cancer tissues compared with adjacent normal tissues. Higher EN2 expression was significantly associated with poorer survival rate. Knockdown of EN2 markedly inhibited proliferation and migration capacities of SW480 cells in vitro, and suppressed tumorigenicity in vivo. Mechanistically, Chemokine ligand 20 (CCL20), a member of the C-C motif chemokine subfamily, was identified as a direct target gene of EN2 in CRC. CCL20 expression was positively correlated with EN2 expression in CRC tissues. Moreover, EN2 promoted the proliferation and migration of CRC cells by regulating the expression of CCL20 in vitro. These results suggest that EN2 plays a critical role in the CRC tumor progression and may serve as a potential target for CRC prevention and therapy.
\end{abstract}

\section{Introduction}

Colorectal cancer (CRC) is the fourth most common cause for cancer-related death worldwide ${ }^{1}$. Despite improvements in the therapeutic strategies including surgery and chemoradiotherapy, CRC patients still have extremely poor prognosis. Thus, there is an urgent need to identify new functional genes and biomarkers in the pathogenesis of CRC for developing effective treatment strategies.

Homeobox-containing genes are important transcription factors, including HOX, EMX, PAX, engrailed (EN) and so on, which play key roles in both embryonic development and oncogenesis ${ }^{2,3}$. Engrailed, a subgroup of homeodomain-containing family, functions in a variety of

\footnotetext{
Correspondence: Guang Shu (shuguang78@csu.edu.cn) or

Gang Yin (gangyin@csu.edu.cn)

'Department of Pathology, Xiangya Hospital, School of Basic Medical Sciences, Central South University, Changsha, China

${ }^{2}$ School of Basic Medical Sciences, Central South University, Changsha, Hunan Province, China

Full list of author information is available at the end of the article

These authors contributed equally: Yimin Li, Jiaxin Liu.

Edited by $\mathrm{G}$ Dewson
}

animal development processes ${ }^{4,5}$. The result of Bioinformatics analysis implied that EN2, a member of the engrailed homeobox family of homo sapiens, was overexpressed in CRC. Previous research has shown the abnormal expression of EN2 in a wide variety of tumor. For instance, Gómez-Gómez E et al. demonstrated that EN2 was overexpressed in prostate cancer tissues. The treatment of EN2 increased cell proliferation, migration, and PSA secretion ${ }^{6}$. EN2 was also discovered to be expressed ectopically in human breast cancer tissues and cell lines and to promote the adenocarcinoma formation ${ }^{7}$. A recent study reported that EN2 was elevated in serous ovarian tumors compared with the normal ovary ${ }^{8}$. However, the expression and the clinical significance of EN2 in CRC remain elusive so far.

CCL20 plays a crucial role in colorectal cancer and thyroid cancer $^{9-11}$. A large number of literatures provided evidence that CCL20 was significantly increased in CRC ${ }^{12}$. CCL20 regulated CRC proliferation and metastasis by resulting in phosphorylation of p130cas and stimulating ERK-MAP kinase and Akt pathways ${ }^{13,14}$. The regulatory mechanism of CCL20 expression in CRC is not clearly

\section{(c) The Author(s) 2020}

(c) (i) Open Access This article is licensed under a Creative Commons Attribution 4.0 International License, which permits use, sharing, adaptation, distribution and reproduction cc) in any medium or format, as long as you give appropriate credit to the original author(s) and the source, provide a link to the Creative Commons license, and indicate if changes were made. The images or other third party material in this article are included in the article's Creative Commons license, unless indicated otherwise in a credit line to the material. If material is not included in the article's Creative Commons license and your intended use is not permitted by statutory regulation or exceeds the permitted use, you will need to obtain permission directly from the copyright holder. To view a copy of this license, visit http://creativecommons.org/licenses/by/4.0/. 
understood. In the present study, we tested the expression of EN2 in colorectal cancer and paired adjacent normal tissues, and then discovered that EN2 was upregulated in the CRC. Moreover, we showed the strong correlation between the high expression of EN2 and the poor survival rate. Through in vitro and in vivo assays, we demonstrated that EN2 significantly enhanced the proliferation and migration of CRC cells by regulating the expression of CCL20.

\section{Materials and methods \\ Bioinformatics analysis}

The CRC cohorts, GSE9348 ${ }^{15}$, was downloaded from the Gene Expression Omnibus (GEO) database (http:// www.ncbi.nlm.nih.gov/geo/). Quantile normalization and $\log 2$ transformation were employed for the expression profiles with R software (http://www.bioconductor.org/). GSE9348 has 70 colorectal cancer samples and 12 normal colorectal samples, which were used to detect the expression of EN1 and EN2 in CRC. Next, we verified the expression of EN2 in colorectal tissues in GTEx (507 normal tissue samples) and TCGA databases (568 tumor samples and 44 normal tissue samples). The expression of EN2 was evaluated by means and standard error of the mean (SEM) with Graphpad Prism Software 8.0. The power of EN2 to differentiate between colorectal cancer and normal tissues was evaluated according to ROC curves. Co-expression gene screening for EN2 in CRC patients was performed by cor function in the $\mathrm{R}$ platform. The screening criteria were as follows: $P<0.05$, and | Pearson correlation coefficient $\mid \geq 0.3$. To determine how EN2 affected the prognosis of CRC patients, we performed Kyoto Encyclopedia of Genes and Genomes (KEGG) analysis of EN2 co-expressed genes. These gene functional enrichment analyses were performed using the DAVID (https://david.ncifcrf.gov/).

\section{Cell lines and cell culture}

HEK-293T and CRC cell lines (SW480 and SW620) were purchased from American Type Culture Collection (ATCC; http://www.atcc.org/). HCT8 cell lines were friendly provided by Professor Wancai Yang (Institute of Precision Medicine, Jining Medical University). The normal colon epithelial cell line 8401 were kindly provided by Professor Lunquan Sun (Xiangya Hospital, Central South University). All cell lines were cultured by RPMI-1640 (Biological industries, Kibbutz belt haemek, Israel) with $10 \%$ fetal bovine serum (FBS; Biological industries, Kibbutz belt haemek, Israel) at $37^{\circ} \mathrm{C}$ with $5 \% \mathrm{CO}_{2}$.

\section{Patients and samples}

The CRC samples and paired normal tissues were obtained from Xiangya Hospital of Central South University. The patients were informed and signed the informed consent. This work was approved by the Ethics Committee of Xiangya Hospital. The clinical pathological parameters of CRC patients were shown in Table 1.

\section{Plasmids, transfection, and infection}

For overexpression of EN2 in CRC cells, the full-length EN2 cDNA was amplified from SW480 cell and then inserted into pcDNA 3.1 vector. The promoter regions of CCL20 and the corresponding mutant were cloned into the pGL3-basic vector. The PLKO.1 vector was used to clone the shRNAs targeting EN2. The pcDNA 3.1-EN2Flag and pCDH-CMV-MCS-EF1-CCL20 plasmids were obtained from CUSABIO (Wuhan, China). To silence EN2, two small interfering RNAs (siRNAs) were purchased from RiboBio (Guangzhou, China). The siRNAs sequences targeting EN2 were as follows: EN2 si\#1: AGTTCCAGACCAACAGGTA; EN2 si\#2: ACCCGAA CAAAGAGGACAA. Transient transfection of siRNA or plasmid was performed by using a standard protocol from the jetPRIME DNA \& siRNA Transfection Reagent (PolyPlus-transfection, France). The transfected cells were harvested after $48 \mathrm{~h}$. The overexpression and the silence efficiency were determined by Western blot. Stable cell lines expressing EN2 shRNA were generated via retroviral infection. Briefly, independent shRNAs against EN2 were constructed using a pLKO.1 vector. The HEK-293T cells were transfected with PLKO.1 based shRNAs, pREV, pGag, and pVSVG at the ratio of 2:2:2:1. The virus particles were collected $48 \mathrm{~h}$ after transfection. The SW480 cells were infected with recombinant lentivirus transducing units using $1 \mu \mathrm{g} / \mathrm{ml}$ polybrene (Sigma-Aldrich, St. Louis, MO), and the stable cell lines were selected for 14 days with $1 \mu \mathrm{g} / \mathrm{ml}$ puromycin.

\section{Cell proliferation assays}

Cell proliferation was tested by Cell Counting Kit- 8 (CCK8) assay, Colony formation assay and EDU assay. CCK8 assay was carried out as previously described ${ }^{16}$. For colony formation assay, 300 cells were seeded in 12-well plates for 10 days. Cell colony was fixed and stained with crystal violet and the numbers of colonies were estimated using the ImageJ. EdU (RiboBio, Guangzhou, China) assay was performed according to a standard protocol as described. All the experiments were repeated at least three times.

\section{Cell migration assays}

The migration ability of the cells was determined by transwell assay and wound healing assay, which were carried out as previously described ${ }^{16}$. For transwell assay, a total of $1 \times 10^{6}$ transfected cells were seeded into the top chamber of a 24-well polycarbonate transwell filter $(8 \mu \mathrm{m}$ pore size, Corning Incorporated, USA). Media containing $20 \%$ FBS was placed in the lower chamber. After 
Table 1 Correlations between EN2 expression and clinicopathologic features in 165 colorectal cancer patients.

\begin{tabular}{|c|c|c|c|c|c|}
\hline \multirow[t]{2}{*}{ Clinicopathological feature } & \multirow[b]{2}{*}{ Total (165) } & \multicolumn{2}{|c|}{ Expression of EN2 } & \multirow[b]{2}{*}{$x^{2}$} & \multirow[b]{2}{*}{$P$-value } \\
\hline & & Low $(n=64)$ & High $(n=101)$ & & \\
\hline Age (years) & & & & 0.984 & 0.321 \\
\hline$\leq 65$ & 90 & 38 & 52 & & \\
\hline$>65$ & 75 & 26 & 49 & & \\
\hline Gender & & & & 2.457 & 0.117 \\
\hline Male & 96 & 32 & 64 & & \\
\hline Female & 69 & 32 & 37 & & \\
\hline Tumor location & & & & 0.011 & 0.915 \\
\hline Colon & 123 & 48 & 75 & & \\
\hline Rctum & 42 & 16 & 26 & & \\
\hline Tumor size & & & & 10.531 & 0.001 \\
\hline$\leq 5 \mathrm{~cm}$ & 77 & 40 & 37 & & \\
\hline$>5 \mathrm{~cm}$ & 88 & 24 & 64 & & \\
\hline Histology grade & & & & 9.726 & 0.002 \\
\hline Well & 78 & 40 & 38 & & \\
\hline Moderate/poor & 87 & 24 & 63 & & \\
\hline TNM stage & & & & 19.498 & $<0.001$ \\
\hline |//I & 61 & 37 & 24 & & \\
\hline III/IV & 104 & 27 & 77 & & \\
\hline
\end{tabular}

The bold number represents the $P$-values with significant differences.

incubation for $12 \mathrm{~h}$, the migration cells on the lower surface were fixed with $4 \%$ paraformaldehyde and stained with $5 \%$ crystal violet. The numbers of crystal violet stained cells in five random fields were counted using an inverted microscope (Olympus, Tokyo, Japan). For wound healing assay, cells were seeded in 6-well plates, and grown to $100 \%$ confluency. A $10-\mu$ l pipette tip was applied to wound the cell monolayer. Subsequently, the cells were incubated in free-serum medium and cultured for $48 \mathrm{~h}$. Wound closure was photographed using an inverted microscope.

\section{RNA extraction and real-time PCR}

All RNA was extracted with Trizol reagent (Vazyme, Nanjing,China), and the first-strand cDNA was inversely transcribed from total RNA $(2 \mu \mathrm{g})$ using GoScript Reverse Transcription System (Promega, Madison, WI, USA). Then, qRT-PCR was performed to analyze cDNA using GoTaq qPCR Master Mix (Promega, Madison, WI, USA) on an ABI Prism 700 thermal cycler (Applied Biosystems, Foster City, CA, USA). Specific steps for qRT-PCR have been shown in our previous studies ${ }^{16}$. Ninety-six-well PCR Plates were used for all tests (NEST Biotechnology,
No.402301, China). The qRT-PCR conditions for glyceraldehyde-3-phosphate dehydrogenase (GAPDH), EN2, and CCL20 were $95^{\circ} \mathrm{C} 10 \mathrm{~min}$, followed by 40 cycles of $95^{\circ} \mathrm{C} 15 \mathrm{~s}, 60^{\circ} \mathrm{C} 60 \mathrm{~s}$. All experiments were carried out in triplicate, and the relative expression of interest genes was normalized to the expression of GAPDH, using $2^{-\Delta \Delta c t}$ method. Primer sequences were as follows: EN2 (forward primer: AGGAGCTGAGCCTCAACGAGTC; reserve primer: CTTGGCTGTGGTGGAGTGGTTG); CCL20 (forward primer: TGCTGTACCAAGAGTTTGCTC; reserve primer: CGCACACAGACAACTTTTTCTTT); GAPDH (forward primer: CTGGGCTACACTGAGCACC; reserve primer: AAGTGGTCGTTGAGGGCAATG).

\section{Western blot}

Total protein was extracted with RIPA buffer containing protease inhibitors and the concentration was measured with the BCA kit. SDS-PAGE was performed with $30 \mu \mathrm{g}$ total protein. The methods can be found in early study ${ }^{16}$. Primary antibodies included anti-EN2 (1:500, ab45867, abcam, UK), anti-Lamin B1 (1:1000, 12987-1-AP, proteintech, China), and anti-GAPDH (1:5000, 60004-1-Ig, proteintech, China). 


\section{Enzyme-linked immunosorbent assay (ELISA)}

The supernatant of treated cells was collected and stored at $-80^{\circ} \mathrm{C}$. CCL20 cytokine levels were measured through the highly sensitive ELISA kits (4 A biotech, CHE0061, China). The follow-up steps were carried out according to manufacturer's instructions.

\section{Nuclear and cytoplasmic fractionation assay}

Nuclear and cytosolic protein were extracted by Nuclear and Cytoplasmic Protein Extraction Kit (Beyotime, P0028, China). The follow-up steps were carried out according to manufacturer's instructions. For the cytoplasmic extract, SW480 cells were lysed in P0028-1 and P0028-2 buffer. Nuclei were then resuspended in P0028-3 buffer.

\section{Immunohistochemical staining}

The protein expression of EN2 and CCL20 were determined by immunohistochemical (IHC) carried out as previously described ${ }^{16}$. The primary antibodies were as follows: anti-EN2 (1:100, MAB2600, USA), anti-CCL20 (1:50, CSB-PA389153, China). Five areas of positive stains were selected to estimate at high $(\times 200)$ magnification using light microscopy by two pathologists who were blinded to the clinicopathological data. The staining index (SI) was calculated as tissue staining intensity and percentage. The percentage of cells was graded as follows: 1 (0-25\%), 2 (26-50\%), $3(51-75 \%)$, or $4(>75 \%)$. Staining intensity was scored as follows: 0 (negative), 1 (weak), 2 (moderate), and 3 (strong); the percentage of cells was scored in the following four categories: 1 (0-25\%), 2 $(26-50 \%), 3(51-75 \%)$, or $4(>75 \%)$. SI $=$ staining percentage $\times$ intensity. Samples with $\mathrm{SI} \geq 6$ were determined as high expression, and those with SI $<6$ were determined as low expression.

\section{Luciferase reporter assay}

HEK-293T, SW480, and HCT8 cells were inoculated into a 24-well plate with a density of $1 \times 10^{5} \mathrm{cell} / \mathrm{ml}$, and when the confluence reached $70 \%$, the plasmids were contransfected with the jetPRIME ${ }^{\circledR}$ DNA \& siRNA Transfection Reagent (PolyPlus-transfection, France). After $48 \mathrm{~h}$, the cells were lysis and measured by Dual-Glo luciferase assay (Promega, Madison, WI, USA) and the luciferase activity was obtained by normalizing with Renilla for each sample. Each experiment was repeated three times.

\section{Chromatin immunoprecipitation (ChIP)}

ChIP assay was performed using ChIP kit (Santa Cruz Biotechnology, CA, USA) according to the manufacturer's instructions. First, EN2-Flag was overexpressed in HCT8 cells. Cells were crosslinked in $1 \%$ formaldehyde for $10 \mathrm{~min}$ at $37^{\circ} \mathrm{C}$ and then lysed in SDS buffer. Sonication was used to fragment the DNA. ChIP for EN2 was performed using a Flag antibody (CST, \#14793 S). Eluted DNA fragments were analyzed by qPCR using the specific primers are listed as follows: CCL20-P1 (forward primer: AGTCCTTCGATGCCTGCTAAG; reserve primer: ACC GTGCCCAGCAGAATAG), CCL20-P2 (forward primer: CTATTCTGCTGGGCACGGT; reserve primer: GCCTC AGCCTCCTGAGTAG), CCL20-P3 (forward primer: GTCTGATATAGGCATCACCAACTCC; reserve primer: CCTATCAGCAGTAGCTAGTCAGC).

\section{Animal study}

All animal protocols were approved by the Institutional Animal Care and Use Committee of Central South University (Changsha, China) as previously described ${ }^{16}$. Stable cells $\left(3 \times 10^{6}\right)$ of EN2 knocked down were subcutaneously injected into the upper limb flank of 5 -weekold BALB/c (nu/nu) nude mice ( $n=5 /$ group). The tumor size was measured and recorded. All tumor grafts were excised, weighed, fixed in formalin and embedded in paraffin, of which sections were stained with $\mathrm{HE}$ and IHC.

\section{Statistical analysis}

All quantitative data were presented as the mean \pm standard error of the mean (SEM) deviation from at least three independent experiments. Statistical analysis was carried out using SPSS version 20.0 and Graphpad Prism Software 8.0. The significance of difference between groups was analyzed by Student's $t$-test. Survival curves were analyzed by the Kaplan-Meier analysis with log-rank test. Receiver operating characteristic (ROC) curve analysis was done by RStudio. P-value of less than 0.05 considered to be significant.

\section{Results}

EN2 is upregulated in colorectal cancer and related to poor prognosis of patients

The homo sapiens Engrailed-1 (EN1) and Engrailed-2 (EN2) genes encode homeobox-containing transcription factors that are the homologs of engrailed gene. To identify critical engrailed genes that contribute to the colorectal cancer tumorigenesis, we analyzed the mRNA expression of EN1 and EN2 in GSE9348, in which EN2 was significantly upregulated in CRC (Fig. 1a). Next, we would like to validate whether the expression of EN2 from GSE9348 is consistent with the expression in colorectal tissues from TCGA and GTEx. As expected, the results were so (Fig. 1b). Furthermore, we verified the expression of EN2 in CRC tissues and cell lines. The expression of EN2 was examined by qRT-PCR and Western blot in the colorectal cancer tissues and adjacent normal tissues, together with the CRC cell lines (HCT8, SW620, and SW480) and human intestinal epithelial cell line (8401). As is shown in Fig. 1c, d, both the protein and mRNA levels of EN2 were significantly upregulated in colorectal 


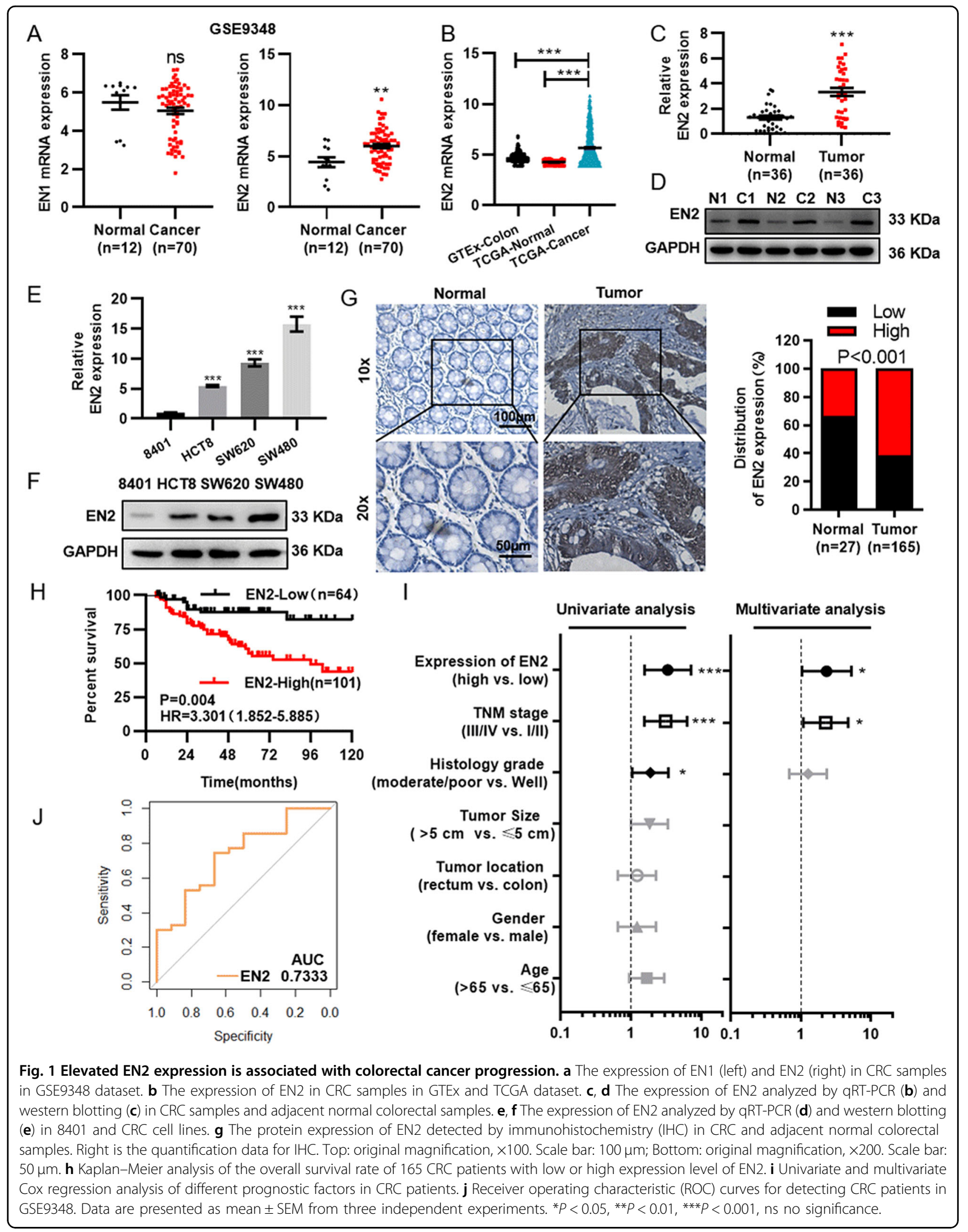


cancer tissues. All CRC cell lines showed significant high expressions of EN2 compared to 8401 cells (Fig. 1e, f).

To make a further assessment of the correlation between the expression level of EN2 and the clinicopathological features of CRC, IHC was used to detect the protein level of EN2 in paraffin embedded tissues. The results showed that EN2 expression was significantly higher in tumor tissues compared to the normal tissues (Fig. 1g). The correlation between EN2 protein and the clinicopathological characteristics of patients was observed, which revealed that the expression level of EN2 was significantly associated with the tumor size, histological grade and TNM stage (Table 1). Further, with the aim of exploring whether EN2 could be a potential prognostic factor for colorectal cancer, we performed Kaplan-Meier survival analysis on the basis of the EN2 expression levels of 165 colorectal cancer patients. The results suggested that the CRC patients with the low EN2 expression had the higher overall survival rate than those with relatively high expression (Log-Rank, $P<0.05$ ) (Fig. 1h).

Meanwhile, univariate and multivariate Cox regression analysis was performed on the paraffin embedded tissues to assess the correlation between EN2 and survival in the presence of clinicopathological characteristics. The analysis showed that the expression of EN2 was an independent prognostic factor for patients' survival, in addition to TNM stage (Fig. 1i). Moreover, receiver operating characteristic (ROC) curve analysis was also performed. We found that the expression of EN2 was enough to distinguish CRC cancer patients' tissues from normal ones (Fig. 1j).

\section{EN2 knockdown restrains proliferation and migration of CRC cells in vitro}

To further explore the role of EN2 in CRC and the mechanisms of its oncogenic function, we screened the EN2 co-expressed genes from GSE9348 $(|\mathrm{R}| \geq 0.3)$ and then performed KEGG pathway analysis on those genes, among which some positively correlated with EN2 enriched in cell cycle, RNA transport and oocyte meiosis, while others negatively correlated with it enriched in metabolic pathways, biosynthesis of antibiotics and fatty acid degradation (Fig. 2a, b). Therefore, the results from this study indicated that EN2 was related with a range of cancer-associated pathways. Also, the results of IHC revealed that there was the correlation between EN2 and the tumor size, suggesting that EN2 might be involved in the regulation of proliferation (Table 1). Next, we knocked down the EN2 in the SW480 cell line, which had high EN2 expression, whereas overexpressed the EN2 in the HCT8 cell line, which had relative low expression (Fig. 2c). To verify the effect of EN2, we detected the cell proliferation and viability of colorectal cancer cells by
CCK8, EDU, and cell clone-formation assay. Further, silencing EN2 in CRC inhabited cell growth distinctly, while overexpressing EN2 promoted the growth (Fig. 2d-f). To further study the effect of EN2 on cell migration, we performed the transwell assay and wound healing test and discovered the silence of EN2 significantly repressed CRC cell migration, whereas the opposite results were observed in CRC cells with the overexpression of EN2 (Fig. 2g, h).

\section{EN2 knockdown prohibits tumorigenicity in vivo}

To further verify the effect of EN2 on tumorigenesis in vivo, we established the subcutaneous xenograft tumor models using SW480 cells with or without EN2 knockdown. As shown in Fig. 3a, we constructed shRNA vectors of EN2 and transfected them into SW480 cells to establish a cell line expressing low level of EN2 stably (Fig. 3a, b). Then, these cells were subcutaneously implanted into the nude mice, and tumor growth was subsequently quantified. The results revealed that EN2 knockdown significantly reduced the tumor growth (Fig. 3c), as well as resulted in the decrease of both volume and weight of tumors (Fig. 3d, e). Further analysis of xenografted CRC tissues by immunohistochemical staining confirmed that the protein level of EN2 decreased in the EN2 knockdown group (Fig. 3f). Collectively, these results indicated that EN2 played a critical role in CRC cell growth in vivo.

\section{EN2 is mainly localized in the nucleus as a transcription factor}

Previous studies had revealed that EN2 was located in the cytoplasm of breast and prostatic cancer ${ }^{7,17}$. However, our results showed EN2 was mainly located in the nucleus of colorectal cells (Fig. 1f). Nuclear and cytoplasmic fractionation assay confirmed the nuclear distribution of EN2 in SW480 cells (Fig. 4a). Additionally, we also transfected EN2-Flag into HCT8 cells (Fig. 4b). Western blot further confirmed the nuclear distribution of EN2Flag. Combined, the results raised the conjecture that EN2 might be a transcription factor involving in the progression of colorectal cancer.

To investigate the molecular mechanism of EN2 in colorectal cancer, we explored the gene expression profiles change of CRC cells after knocking down EN2 through RNA sequencing (Fig. 4c). Gene ontology (GO) and KEGG pathway enrichment analyses were applied to analysis the differently expressed genes (DEGs). In the biological process group, DEGs were mainly enriched in cell response to lipopolysaccharide, wound healing and neutrophil chemotaxis. Cell component analysis revealed that DEGs were mainly gathered at the cell surface, extracellular region, and intracellular. In addition, the molecular function analysis showed that DEGs were mainly enriched in the sequence-specific DNA binding, 


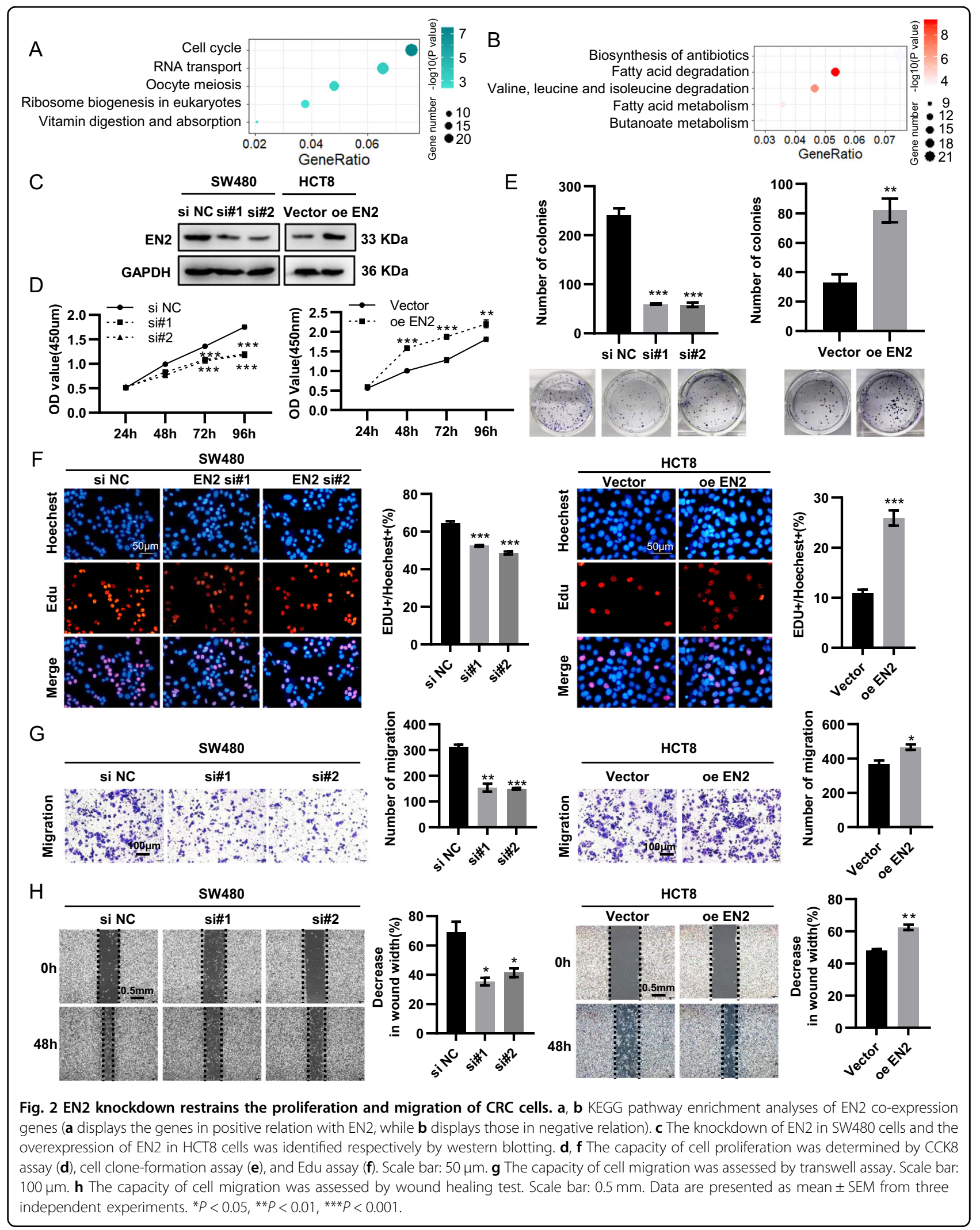


A
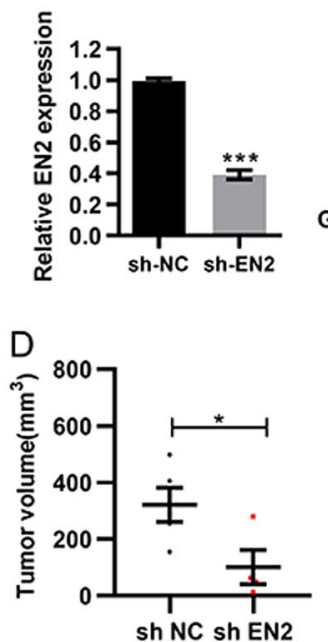

B

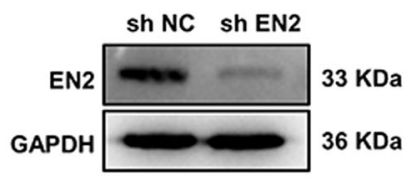

$\mathrm{E}$

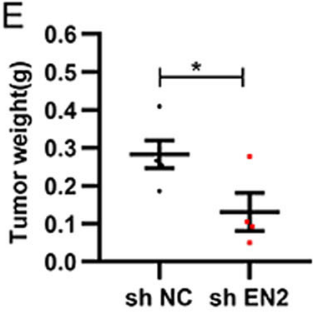

C
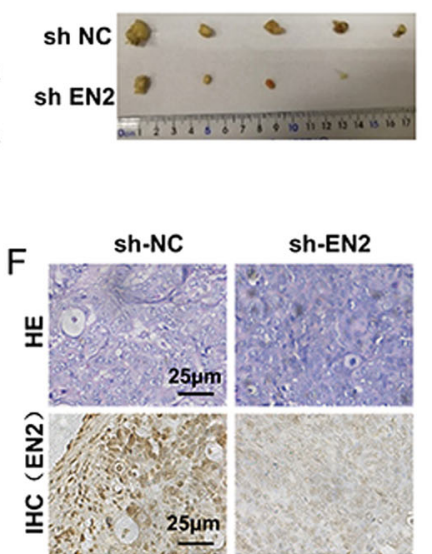

Fig. 3 EN2 knockdown restrains tumor growth in vivo. $\mathbf{a}$, b The knockdown of EN2 in SW480 cells were identified by qRT-PCR (a) and western blotting (b). c The tumor growth curves of shRNA-EN2 and shRNA-NC groups in the xenograft mouse model. $\mathbf{d}$, e The tumor volume (d) and weight (e) of nude mice were measured. $\mathbf{f}$ Representative images of H\&E staining and IHC staining in subcutaneous tumors from nude mice. Scale bar: $25 \mu \mathrm{m}$. Data are presented as mean \pm SEM. ${ }^{*} P<0.05$.

the growth factor activity and the cytokine activity (Supplementary Fig. 1A). KEGG pathway showed that DEG gathered at TNF pathway, cytokine-cytokine receptor, HTLV-1 signing pathway and so on (Supplementary Fig. 1B).

\section{CCL20 acts as the target gene of EN2}

Among these DEGs, CCL20 expression exhibited the highest fold change in si-EN2 group and thus CCL20 was selected for further experimental validation (Fig. 4c). The knockdown of EN2 notably suppressed the expression of CCL20, which was determined by qRT-PCR and ELISA (Fig. 4d, e). To confirm the regulatory relationship between EN2 and CCL20 in CRC, we performed JASPAR database analysis and discovered that the promoter of CCL20 gene contained the EN2 binding sites (Fig. 4f). Next, we identified the binding sites of EN2 on the CCL20 promoter using ChIP assay. Three pairs of primers according to the predicted binding sites were used for qPCR after ChIP (Fig. 4g). ChIP assay demonstrated that EN2 interacted physiologically with the P1 $(-1456$ to $-1447 \mathrm{bp}$ ) region on the promoter of the CCL20 (Fig. 4h). Furthermore, we generated luciferase reporter plasmids: CCL20-P-WT, containing $-2000 \mathrm{bp}$ upstream of the transcriptional start site, and CCL20-P-MUT, containing a mutated in P1 ( -1456 to -1447 bp) region (Fig. 4i). Luciferase reporter assay confirmed that EN2 prominently increased luciferase activity in CCL20-P-WT group not in CCL20-P-MUT in $293 \mathrm{~T}$ cells (Fig. 4i). Furthermore, the overexpression of EN2 increased the CCL20 promoterdriven reporter activity in HCT8 cells, while the silencing of EN2 had the opposite results (Fig. 4j, k). Next, we wondered whether the EN2-CCL20 axis is clinically relevant. The results of IHC analysis showed that EN2 protein expression was correlated positively with the level of CCL20 (Fig. 4l). A positive correlation between EN2 and CCL20 mRNA expression was verified in GSE9348 and TCGA databases (Fig. 4m). The AUC of EN2 and EN2 combined with CCL20 were 0.733 and 0.895 . This analysis indicated that EN2 combined with CCL20 for the CRC diagnosis was better than EN2 alone (Fig. 4n). Taken together, our results indicated that CCL20 was a downstream target of EN2 in CRC cells.

\section{EN2 promotes CRC proliferation and migration via upregulating $\mathrm{CCL} 20$}

Since CCL20 acted as a direct target gene of EN2, we then performed functional recovery experiments to determine whether EN2 exerts functions by regulating CCL20 expression in CRC (Fig. 5a). SW480 cells with EN2 knocked down were transfected with OE-CCL20 and then the cell proliferation and migration abilities were measured. The results showed that the restrained proliferation and migration caused by the knockdown of EN2 could be reversed when CCL20 was upregulated (Fig. 5b-e). These data confirmed that EN2 promoted CRC proliferation and migration by regulating CCL20 expression.

\section{Discussion}

Recent studies have shown that homeobox-containing genes, including HOX, EMX, PAX, EN, and so on, as key regulators contribute to the development and progression 


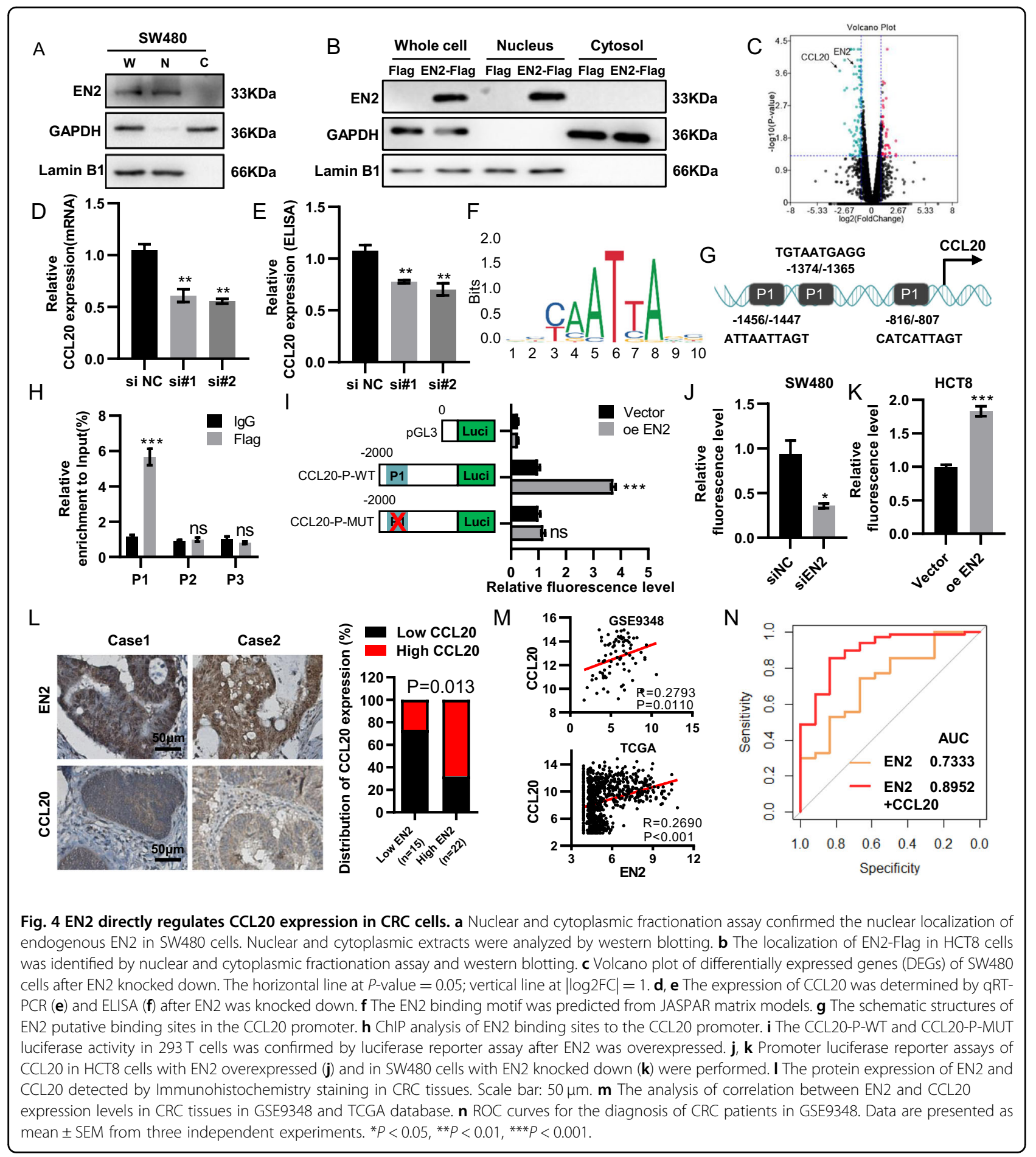

of a variety of human cancers ${ }^{3,18,19}$. As the human engrailed homolog, EN2 has been reported to correlate closely with human cancers such as prostate, breast, bladder, ovarian cancer, and non -small cell lung cancer ${ }^{6-8,20,21}$. However, it remains unclear whether EN2 has any role in colorectal cancer. Our study found EN2 expression level was higher in CRC tissues than that in tumor adjacent tissues on the basis of GES9478, TCGA, and our CRC specimens. The high expression of EN2 is significantly correlated with tumor size, histological grade, advanced TNM stage as well as poor survival of patients. We also found that EN2 knockdown could suppress CRC cell 


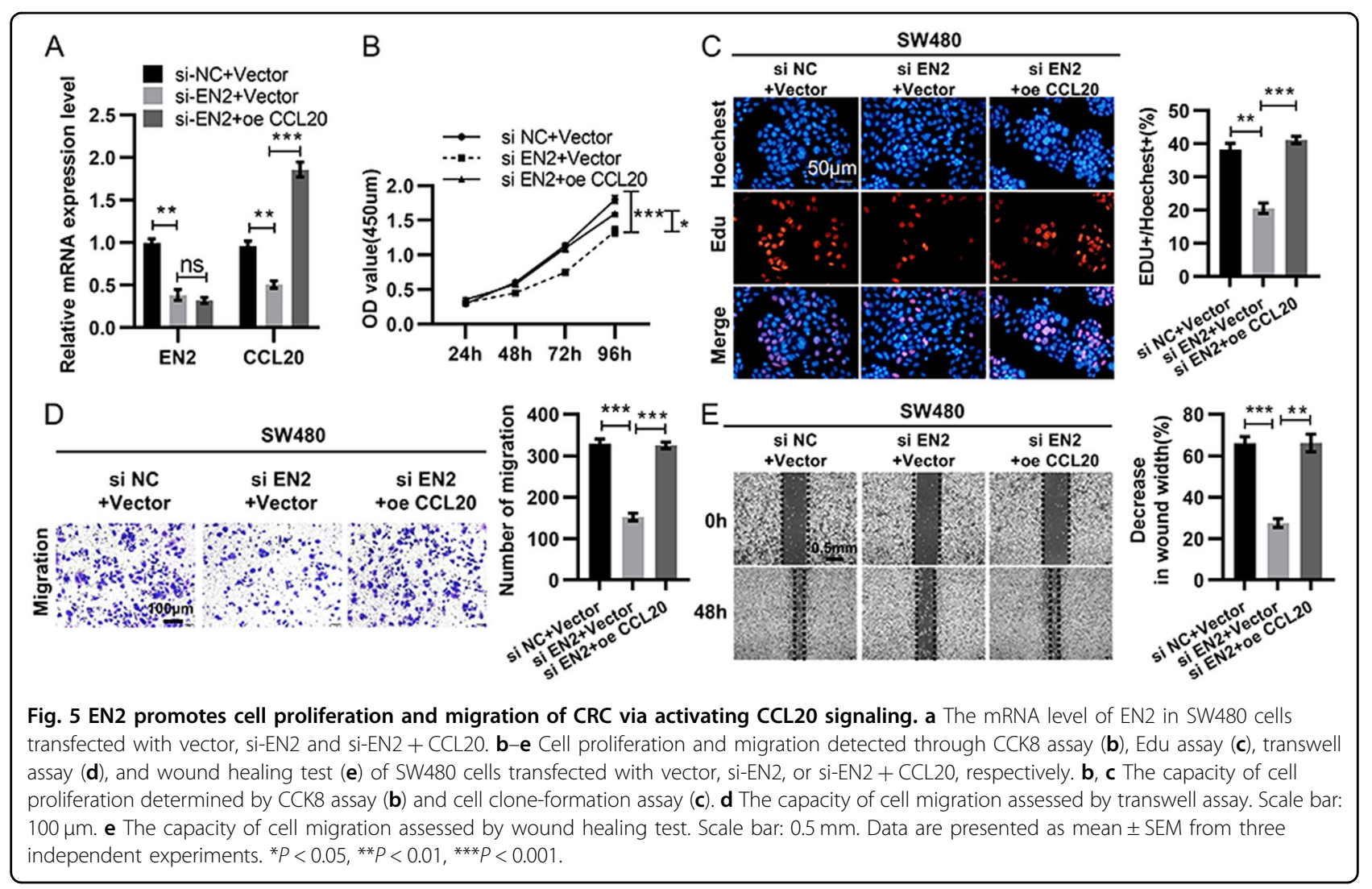

proliferation and migration in vitro and in vivo. These results indicate that EN2 may be an independent diagnostic and prognostic marker for CRC patients.

Pre-existing research had revealed that EN2 was located in the cytoplasm of breast and prostate cancer ${ }^{7,17}$. In this study, we showed that EN2 was mainly located in CRC cell nucleus, which means that EN2 act as a transcription factor to activate the expression of downstream genes in CRC. We further investigated the potential mechanism of EN2 in tumorigenesis of CRC by RNA sequencing, which revealed that EN2 might regulate wound healing, growth factor activity, cytokine activity, and TNF pathway, confirming that EN2 was crucial in CRC tumorigenesis. Next, we used the ChIP and luciferase assay to explore a direct binding site of the EN2 on the promoter region of CCL20, which provides evidence that CCL20 is regulated by EN2 transcription. Furthermore, the positive correlation between CCL20 and EN2 was verified in both mRNA and protein levels in CRC tissues.

CCL20 functions as a sort of chemokine existing in a variety of human tissues ${ }^{8,22}$. Also, CCL20 is highly expressed in colorectal cancer cells and contributes to cancer progression ${ }^{23,24}$. For example, in CRC, Yu X et al. and Cheng et al. demonstrated that CCL20 overexpression could promote cell proliferation and migration in $\mathrm{CRC}^{25}$. Additionally, Wang D et al. reported that the high level of CCL20 was closely associated with the poor survival of CRC patients. CRC cell-secreted CCL20 can recruit Tregs that further enhanced the chemoresistance of CRC cells to 5-FU' ${ }^{26}$. Besides, CCL20 could influence the microenvironment via $\mathrm{B}$ and $\mathrm{T}$ cells, thus affecting CRC progression ${ }^{27}$. In our study, we discovered that EN2 was able to positively regulate the expression of CCL20 in CRC. Moreover, the restrained cell proliferation and migration caused by knockdown of EN2 could be reversed by upregulating CCL20. These results indicate EN2 exerts its function, at least in part, through regulating CCL20 expression.

In conclusion, our study indicates that EN2 is frequently upregulated in CRC tissues and associated with poor prognosis and thus can be a promising diagnostic and prognostic marker for CRC patients. In mechanism, EN2 promotes CRC cell proliferation and migration, at least partially, by regulating CCL20 expression. Taken together, these findings suggest that EN2 is an oncogene in CRC and may serve as a promising target for CRC prevention and therapy.

\section{Acknowledgements}

This work was supported by grants from the National Key R\&D Program of China, Stem Cell and Translational Research (No. 2016YFA0102000); The National Natural Science Foundation of China (No. 81572900); The 
Fundamental Research Funds for the Central Universities of Central South University [No. 2018zzts232].

\section{Author details}

'Department of Pathology, Xiangya Hospital, School of Basic Medical Sciences, Central South University, Changsha, China. ${ }^{2}$ School of Basic Medical Sciences, Central South University, Changsha, Hunan Province, China. ${ }^{3}$ China-Africa Research Center of Infectious Diseases, School of Basic Medical Sciences, Central South University, Changsha, Hunan Province, China

\section{Conflict of interest}

The authors declare that they have no conflict of interest.

\section{Publisher's note}

Springer Nature remains neutral with regard to jurisdictional claims in published maps and institutional affiliations.

Supplementary Information accompanies this paper at (https://doi.org/ 10.1038/s41419-020-02804-3).

Received: 26 February 2020 Revised: 7 July 2020 Accepted: 13 July 2020 Published online: 30 July 2020

\section{References}

1. Bray, F. et al. Global cancer statistics 2018: GLOBOCAN estimates of incidence and mortality worldwide for 36 cancers in 185 countries. CA Cancer J. Clin. 68 , 394-424 (2018).

2. Morgan, R. \& El-Tanani, M. HOX genes as potential markers of circulating tumour cells. Curr. Mol. Med. 16, 322-327 (2016)

3. Bhatlekar, S., Fields, J. Z. \& Boman, B. M. HOX genes and their role in the development of human cancers. J. Mol. Med. (Berl) 92, 811-823 (2014).

4. Morgan, R. Engrailed: complexity and economy of a multi-functional transcription factor. FEBS Lett. 580, 2531-2533 (2006).

5. Gibert, J. M. The evolution of engrailed genes after duplication and speciation events. Dev. Genes Evol. 212, 307-318 (2002).

6. Gomez-Gomez, E. et al. Oncogenic role of secreted engrailed homeobox 2 (EN2) in prostate cancer. J. Clin. Med. 8, 1400 (2019).

7. Martin, N. L., Saba-El-Leil, M. K., Sadekova, S., Meloche, S. \& Sauvageau, G. EN2 is a candidate oncogene in human breast cancer. Oncogene 24, 6890-6901 (2005).

8. McGrath, S. E. et al. Engrailed-2 (EN2) - a novel biomarker in epithelial ovarian cancer. BMC Cancer 18, 943 (2018).

9. Ghadjar, P., Rubie, C., Aebersold, D. M. \& Keilholz, U. The chemokine CCL20 and its receptor CCR6 in human malignancy with focus on colorectal cancer. Int. J. Cancer 125, 741-745 (2009).

10. Chen, W. et al. CCL20 triggered by chemotherapy hinders the therapeutic efficacy of breast cancer. PLOS Biol. 16, e2005869 (2018).
11. Zeng, W., Chang, H., Ma, M. \& Li, Y. CCL20/CCR6 promotes the invasion and migration of thyroid cancer cells via NF-kappa B signaling-induced MMP-3 production. Exp. Mol. Pathol. 97, 184-190 (2014).

12. Frick, V. O., Rubie, C., Keilholz, U. \& Ghadjar, P. Chemokine/chemokine receptor pair CCL20/CCR6 in human colorectal malignancy: an overview. World J. Gastroenterol. 22, 833-841 (2016).

13. Yang, C. C. et al. Chemokine receptor CCR6 transduces signals that activate p130Cas and alter CAMP-stimulated ion transport in human intestinal epithelial cells. Am. J. Physiol. Cell Physiol. 288, C321-C328 (2005).

14. Brand, S. et al. Cell differentiation dependent expressed CCR6 mediates ERK-1/ 2, SAPKJNK, and Akt signaling resulting in proliferation and migration of colorectal cancer cells. J. Cell Biochem. 97, 709-723 (2006).

15. Hong, Y., Downey, T., Eu, K. W., Koh, P. K. \& Cheah, P. Y. A 'metastasis-prone' signature for early-stage mismatch-repair proficient sporadic colorectal cancer patients and its implications for possible therapeutics. Clin. Exp. Metastasis 27, 83-90 (2010).

16. Li, T. et al. Methylation-mediated repression of MiR-424/503 cluster promotes proliferation and migration of ovarian cancer cells through targeting the hub gene KIF23. Cell Cycle 18, 1601-1618 (2019).

17. Morgan, R. et al. Engrailed-2 (EN2): a tumor specific urinary biomarker for the early diagnosis of prostate cancer. Clin. Cancer Res. 17, 1090-1098 (2011).

18. Scott, M. P., Tamkun, J. W. \& Hartzell, G. W. 3rd The structure and function of the homeodomain. Biochim. Biophys. Acta 989, 25-48 (1989).

19. Catela, C., Shin, M. M., Lee, D. H., Liu, J. P. \& Dasen, J. S. Hox proteins coordinate motor neuron differentiation and connectivity programs through Ret/Gfralpha genes. Cell Rep. 14, 1901-1915 (2016).

20. Li, Y. et al. Repression of engrailed 2 inhibits the proliferation and invasion of human bladder cancer in vitro and in vivo. Oncol. Rep. 33 2319-2330 (2015)

21. Lin, X., Liu, X. \& Gong, C. Expression of engrailed homeobox 2 regulates the proliferation, migration and invasion of non-small cell lung cancer cells. Oncol. Lett. 16, 536-542 (2018).

22. Nagarsheth, N., Wicha, M. S. \& Zou, W. Chemokines in the cancer microenvironment and their relevance in cancer immunotherapy. Nat. Rev. Immunol. 17, 559-572 (2017).

23. Cheng, X. S. et al. CCL20 and CXCL8 synergize to promote progression and poor survival outcome in patients with colorectal cancer by collaborative induction of the epithelial-mesenchymal transition. Cancer Lett. 348, 77-87 (2014).

24. Jin, P. et al. Astrocyte-derived CCL20 reinforces HIF-1-mediated hypoxic responses in glioblastoma by stimulating the CCR6-NF-kappaB signaling pathway. Oncogene 37, 3070-3087 (2018).

25. $\mathrm{Yu}, \mathrm{X}$. et al. The novel long noncoding RNA u50535 promotes colorectal cancer growth and metastasis by regulating CCL20. Cell Death Dis. 9, 751 (2018).

26. Wang, D. et al. Colorectal cancer cell-derived CCL20 recruits regulatory $T$ cells to promote chemoresistance via FOXO1/CEBPB/NF-kappaB signaling. J. Immunother. Cancer 7, 215 (2019).

27. Wunderlich, C. M. et al. Obesity exacerbates colitis-associated cancer via IL-6regulated macrophage polarisation and CCL-20/CCR-6-mediated lymphocyte recruitment. Nat. Commun. 9, 1646 (2018). 